\title{
On the Modes of Polynomials Derived from Nondecreasing Sequences
}

\author{
Donna Q. J. Dou \\ School of Mathematics \\ Jilin University, Changchun 130012, P. R. China \\ qjdou@jlu.edu.cn \\ Arthur L. B. Yang \\ Center for Combinatorics, LPMC-TJKLC \\ Nankai University, Tianjin 300071, P. R. China \\ yang@nankai.edu.cn
}

Submitted: Oct 13, 2010; Accepted: Dec 15, 2010; Published: Jan 5, 2011

Mathematics Subject Classification: 05A20, 33F10

\begin{abstract}
Wang and Yeh proved that if $P(x)$ is a polynomial with nonnegative and nondecreasing coefficients, then $P(x+d)$ is unimodal for any $d>0$. A mode of a unimodal polynomial $f(x)=a_{0}+a_{1} x+\cdots+a_{m} x^{m}$ is an index $k$ such that $a_{k}$ is the maximum coefficient. Suppose that $M_{*}(P, d)$ is the smallest mode of $P(x+d)$, and $M^{*}(P, d)$ the greatest mode. Wang and Yeh conjectured that if $d_{2}>d_{1}>0$, then $M_{*}\left(P, d_{1}\right) \geq M_{*}\left(P, d_{2}\right)$ and $M^{*}\left(P, d_{1}\right) \geq M^{*}\left(P, d_{2}\right)$. We give a proof of this conjecture.
\end{abstract}

Keywords: unimodal polynomials, the smallest mode, the greatest mode.

\section{Introduction}

This paper is concerned with the modes of unimodal polynomials constructed from nonnegative and nondecreasing sequences. Recall that a sequence $\left\{a_{i}\right\}_{0 \leq i \leq m}$ is unimodal if there exists an index $0 \leq k \leq m$ such that

$$
a_{0} \leq \cdots \leq a_{k-1} \leq a_{k} \geq a_{k+1} \geq \cdots \geq a_{m} .
$$

Such an index $k$ is called a mode of the sequence. Note that a mode of a sequence may not be unique. The sequence $\left\{a_{i}\right\}_{0 \leq i \leq m}$ is said to be spiral if

$$
a_{m} \leq a_{0} \leq a_{m-1} \leq a_{1} \leq \cdots \leq a_{\left[\frac{m}{2}\right]}
$$


where $\left[\frac{m}{2}\right]$ stands for the largest integer not exceeding $\frac{m}{2}$. Clearly, the spiral property implies unimodality. We say that a sequence $\left\{a_{i}\right\}_{0 \leq i \leq m}$ is log-concave if for $1 \leq k \leq m-1$,

$$
a_{k}^{2} \geq a_{k+1} a_{k-1}
$$

and it is ratio monotone if

$$
\frac{a_{m}}{a_{0}} \leq \frac{a_{m-1}}{a_{1}} \leq \cdots \leq \frac{a_{m-i}}{a_{i}} \leq \cdots \leq \frac{a_{m-\left[\frac{m-1}{2}\right]}}{a_{\left[\frac{m-1}{2}\right]}} \leq 1
$$

and

$$
\frac{a_{0}}{a_{m-1}} \leq \frac{a_{1}}{a_{m-2}} \leq \cdots \leq \frac{a_{i-1}}{a_{m-i}} \leq \cdots \leq \frac{a_{\left[\frac{m}{2}\right]-1}}{a_{m-\left[\frac{m}{2}\right]}} \leq 1 .
$$

It is easily checked that ratio monotonicity implies both log-concavity and the spiral property.

Let $P(x)=a_{0}+a_{1} x+\cdots+a_{m} x^{m}$ be a polynomial with nonnegative coefficients. We say that $P(x)$ is unimodal if the sequence $\left\{a_{i}\right\}_{0 \leq i \leq m}$ is unimodal. A mode of $\left\{a_{i}\right\}_{0 \leq i \leq m}$ is also called a mode of $P(x)$. Similarly, we say that $P(x)$ is log-concave or ratio monotone if the sequence $\left\{a_{i}\right\}_{0 \leq i \leq m}$ is log-concave or ratio monotone.

Throughout this paper $P(x)$ is assumed to be a polynomial with nonnegative and nondecreasing coefficients. Boros and Moll [2] proved that $P(x+1)$, as a polynomial of $x$, is unimodal. Alvarez et al. [1] showed that $P(x+n)$ is also unimodal for any positive integer $n$, and conjectured that $P(x+d)$ is unimodal for any $d>0$. Wang and Yeh [6] confirmed this conjecture and studied the modes of $P(x+d)$. Llamas and Martínez-Bernal [5] obtained the log-concavity of $P(x+c)$ for $c \geq 1$. Chen, Yang and Zhou [4] showed that $P(x+1)$ is ratio monotone, which leads to an alternative proof of the ratio monotonicity of the Boros-Moll polynomials [3].

Let $M_{*}(P, d)$ and $M^{*}(P, d)$ denote the smallest and the greatest mode of $P(x+d)$ respectively. Our main result is the following theorem, which was conjectured by Wang and Yeh [6].

Theorem 1.1 Suppose that $P(x)$ is a monic polynomial of degree $m \geq 1$ with nonnegative and nondecreasing coefficients. Then for $0<d_{1}<d_{2}$, we have $M_{*}\left(P, d_{1}\right) \geq M_{*}\left(P, d_{2}\right)$ and $M^{*}\left(P, d_{1}\right) \geq M^{*}\left(P, d_{2}\right)$.

From now on, we further assume that $P(x)$ is monic, that is $a_{m}=1$. For $0 \leq k \leq m$, let

$$
b_{k}(x)=\sum_{j=k}^{m}\left(\begin{array}{l}
j \\
k
\end{array}\right) a_{j} x^{j-k} .
$$

Therefore, $b_{k}(x)$ is of degree $m-k$ and $b_{k}(0)=a_{k}$. For $1 \leq k \leq m$, let

$$
f_{k}(x)=b_{k-1}(x)-b_{k}(x)
$$

which is of degree $m-k+1$. Let $f_{k}^{(n)}(x)$ denote the $n$-th derivative of $f_{k}(x)$.

Our proof of Theorem 1.1 relies on the fact that $f_{k}(x)$ has at most one real zero on $(0,+\infty)$. In fact, the derivative $f_{k}^{(n)}(x)$ of order $n \leq m-k$ has the same property. We establish this property by induction on $n$. 


\section{Proof of Theorem 1.1}

To prove Theorem 1.1, we need the following three lemmas.

Lemma 2.1 For any $0 \leq k \leq m$, we have $b_{k}^{\prime}(x)=(k+1) b_{k+1}(x)$.

Proof. Let $B_{j, k}(x)$ denote the summand of $b_{k}(x)$. It is readily checked that

$$
B_{j, k}^{\prime}(x)=(k+1) B_{j, k+1}(x) .
$$

The result immediately follows.

Lemma 2.2 For $n \geq 1$ and $1 \leq k \leq m$, we have

$$
f_{k}^{(n)}(x)=(k+n-1)_{n} b_{k+n-1}(x)-(k+n)_{n} b_{k+n}(x),
$$

where $(m)_{j}=m(m-1) \cdots(m-j+1)$.

Proof. Use induction on $n$. For $n=1$, we have

$$
f_{k}^{(n)}(x)=f_{k}^{\prime}(x)=k b_{k}-(k+1) b_{k+1} .
$$

Assume that the lemma holds for $n=j$, namely,

$$
f_{k}^{(j)}(x)=(k+j-1)_{j} b_{k+j-1}(x)-(k+j)_{j} b_{k+j}(x) .
$$

Therefore,

$$
\begin{aligned}
f_{k}^{(j+1)}(x) & =(k+j-1)_{j} b_{k+j-1}^{\prime}(x)-(k+j)_{j} b_{k+j}^{\prime}(x) \\
& =(k+j)(k+j-1)_{j} b_{k+j}(x)-(k+j+1)(k+j)_{j} b_{k+j+1}(x) \\
& =(k+j)_{j+1} b_{k+j}(x)-(k+j+1)_{j+1} b_{k+j+1}(x) .
\end{aligned}
$$

This completes the proof.

Lemma 2.3 For $1 \leq k \leq m$ and $0 \leq n \leq m-k$, the polynomial $f_{k}^{(n)}(x)$ has at most one real zero on the interval $(0,+\infty)$. In particular, $f_{k}(x)$ has at most one real zero on the interval $(0,+\infty)$.

Proof. Use induction on $n$ from $m-k$ to 0 . First, we consider the case $n=m-k$. Recall that

$$
f_{k}(x)=\sum_{j=k-1}^{m}\left(\begin{array}{c}
j \\
k-1
\end{array}\right) a_{j} x^{j-k+1}-\sum_{j=k}^{m}\left(\begin{array}{l}
j \\
k
\end{array}\right) a_{j} x^{j-k}
$$

Thus $f_{k}(x)$ is a polynomial of degree $m-k+1$. Note that

$$
f_{k}^{(m-k)}(x)=(m-k+1) !\left(\begin{array}{c}
m \\
k-1
\end{array}\right) a_{m} x+\left[\left(\begin{array}{c}
m-1 \\
k-1
\end{array}\right) a_{m-1}-\left(\begin{array}{c}
m \\
k
\end{array}\right) a_{m}\right](m-k) ! .
$$


Clearly, $f_{k}^{(m-k)}(x)$ has at most one real zero $x_{0}$ on $(0,+\infty)$. So the lemma is true for $n=m-k$.

Suppose that the lemma holds for $n=j$, where $m-k \geq j \geq 1$. We proceed to show that $f_{k}^{(j-1)}(x)$ has at most one real zero on $(0,+\infty)$. From the inductive hypothesis it follows that $f_{k}^{(j)}(x)$ has at most one real zero on $(0,+\infty)$. In light of $(2.1)$, it is easy to verify that $f_{k}^{(j)}(+\infty)>0$ and

$$
f_{k}^{(j)}(0)=(k+j-1)_{j} a_{k+j-1}-(k+j){ }_{j} a_{k+j} \leq 0 .
$$

It follows that either the polynomial $f_{k}^{(j-1)}(x)$ is increasing on the entire interval $(0,+\infty)$, or there exists a positive real number $r$ such that $f_{k}^{(j-1)}(x)$ is decreasing on $(0, r]$ and increasing on $(r,+\infty)$. Again by $(2.1)$ we find $f_{k}^{(j-1)}(+\infty)>0$ and

$$
f_{k}^{(j-1)}(0)=(k+j-2)_{j-1} a_{k+j-2}-(k+j-1)_{j-1} a_{k+j-1} \leq 0 .
$$

So we conclude that $f_{k}^{(j-1)}(x)$ has at most one real zero on $(0,+\infty)$. This completes the proof.

Proof of Theorem 1.1. In view of (1.4), we have

$$
P(x+d)=\sum_{k=0}^{m} a_{k}(x+d)^{k}=\sum_{k=0}^{m} b_{k}(d) x^{k} .
$$

Let us first prove that $M^{*}\left(P, d_{1}\right) \geq M^{*}\left(P, d_{2}\right)$. Suppose that $M^{*}\left(P, d_{1}\right)=k$. If $k=m$, then the inequality $M^{*}\left(P, d_{1}\right) \geq M^{*}\left(P, d_{2}\right)$ holds. For the case $0 \leq k<m$, it suffices to verify that $b_{k}\left(d_{2}\right)>b_{k+1}\left(d_{2}\right)$. By Lemma $2.2, f_{k+1}(x)$ has at most one real zero on $(0,+\infty)$. Note that

$$
f_{k+1}(0) \leq 0 \quad \text { and } \quad f_{k+1}(+\infty)>0 .
$$

From $M^{*}\left(P, d_{1}\right)=k$ it follows that $b_{k}\left(d_{1}\right)>b_{k+1}\left(d_{1}\right)$, that is $f_{k+1}\left(d_{1}\right)>0$. Therefore, $f_{k+1}\left(d_{2}\right)>0$, that is, $b_{k}\left(d_{2}\right)>b_{k+1}\left(d_{2}\right)$.

Similarly, it can be seen that $M_{*}\left(P, d_{1}\right) \geq M_{*}\left(P, d_{2}\right)$. Suppose that $M_{*}\left(P, d_{2}\right)=k$. If $k=0$, then we have $M_{*}\left(P, d_{1}\right) \geq M_{*}\left(P, d_{2}\right)$. If $0<k \leq m$, it is necessary to show that $b_{k-1}\left(d_{1}\right)<b_{k}\left(d_{1}\right)$. Again, by Lemma 2.2 , we know that $f_{k}(x)$ has at most one real zero on $(0,+\infty)$. From $M_{*}\left(P, d_{2}\right)=k$, it follows that $b_{k-1}\left(d_{2}\right)<b_{k}\left(d_{2}\right)$, that is $f_{k}\left(d_{2}\right)<0$. By the boundary conditions

$$
f_{k}(0) \leq 0 \quad \text { and } \quad f_{k}(+\infty)>0
$$

we obtain $f_{k}\left(d_{1}\right)<0$, that is $b_{k-1}\left(d_{1}\right)<b_{k}\left(d_{1}\right)$. This completes the proof.

Acknowledgments. This work was supported by the 973 Project, the PCSIRT Project of the Ministry of Education, and the National Science Foundation of China. 


\section{References}

[1] J. Alvarez, M. Amadis, G. Boros, D. Karp, V. H. Moll and L. Rosales, An extension of a criterion for unimodality, Electron. J. Combin. 8 (2001), \#R30.

[2] G. Boros and V. H. Moll, A criterion for unimodality, Electron. J. Combin. 6 (1999), \#R10.

[3] W. Y. C. Chen and E. X. W. Xia, The ratio monotonicity of the Boros-Moll polynomials, Math. Comp. 78 (2009), 2269-2282.

[4] W. Y. C. Chen, A. L. B. Yang and E. L.F. Zhou, Ratio monotonicity of polynomials derived from nondecreasing sequences, Electron. J. Combin. 17 (2010), \#N37.

[5] A. Llamas and J. Martínez-Bernal, Nested log-concavity, Commun. Algebra 38 (2010), 1968-1981.

[6] Y. Wang and Y.-N. Yeh, Proof of a conjecture on unimodality, European J. Combin. 26 (2005), 617-627. 